\title{
Selection of aggressive pathogenic and solopathogenic strains of Ustilago maydis to improve Huitlacoche production
}

\author{
Porfirio Raúl Galicia-García', Hilda Victoria Silva-Rojas", Leopoldo Ernesto Mendoza-Onofre', \\ Hilda Araceli Zavaleta-Mancera² ${ }^{2}$ Leobigildo Córdova-Téllez and Alejandro Espinosa-Calderón ${ }^{3}$
}

Received: March 28, 2016

Accepted: December 5, 2016

\begin{abstract}
Ustilago maydis is a basidiomycete known as the causative agent of 'common smut', worldwide disease of maize that is recognized by the galls it forms, which have considerable potential as a gourmet food. Results of infection are quite variable, even under optimal greenhouse conditions. In order to find pathogenic strains able to be used as a highly infective and stable inoculum for the successful production of galls either in greenhouses or in the field, ears with gall symptoms containing teliospores were recovered from maize plants. The teliospores were suspended in water and plated on nutrient-rich medium. Twenty-six colonies developed, containing three types of yeast-like colonies: saprotrophic, pathogenic, and solopathogenic. DAPI staining confirmed the presence of solopathogenic strains with diploid sporidia. Groups of different mating types were found when pairs of the 26 strains were arranged resembling partial-diallel combinations. Amplification of the partial $b$ locus revealed that the strains found harbor the alleles $b 3$ and $b 4$, allowing the formation in dikaryotic strains of heterodimeric regulatory proteins associated with fungal development and pathogenicity. In this study, we isolated compatible haploid and solopathogenic diploid strains for their high capacity for inducing smut.
\end{abstract}

Keywords: Basidiomycete, maize galls, mating types, molecular diagnosis, smut

\section{Introduction}

The heterothallic basidiomycete, Ustilago maydis, is a fungal pathogen of maize (Zea mays) causing 'common smut' disease (Christensen 1963). Since the mid eighteenth century, studies have shown that its life cycle, mechanisms of dissemination and the availability of resistant maize varieties (Shutleff 1980) generally result in only mild grain yield losses (c. 2 \%) under field conditions due to this pathogen. Although, these losses are small in fractional terms, and can be argued that their commercial value is significant where production levels are especially high as in the USA and China that produce 313 and 192 million tonnes, respectively (FAOSTAT 2013). Maize smut is thus a common disease worldwide, with a significant economic impact on corn sector (Djamei \& Kahmann 2012). U. maydis is also a good model for fungal genetics, and it is considered one of the topten pathogens in molecular plant pathology (Dean et al. 2012).

Mexico is a centre of origin of maize, and so encompasses a very broad genetic diversity. Moreover, the association

\footnotetext{
${ }^{1}$ Colegio de Postgraduados, Producción de Semillas, Campus Montecillo, Km. 36.5 Carretera México-Texcoco, Montecillo, Texcoco, Estado de México, 56230, Mexico

${ }^{2}$ Colegio de Postgraduados, Botánica, Campus Montecillo, Km. 36.5 Carretera México-Texcoco, Montecillo, Texcoco, Estado de México, 56230, Mexico

${ }^{3}$ Instituto Nacional de Investigaciones Forestales, Agrícolas y Pecuarias, Campo Experimental Valle de México, Coatlinchán Texcoco, Estado de México, 56250, Mexico
} 
Porfirio Raúl Galicia-Garcia, Hilda Victoria Silva-Rojas, Leopoldo Ernesto Mendoza-Onofre, Hilda Araceli Zavaleta-Mancera, Leobigildo Córdova-Téllez and Alejandro Espinosa-Calderón

between $U$. maydis and Zea mays and its ancestor, the teosinte, Zea mays sp. parviglumis is well known, as they have co-evolved since pre-Hispanic times (García-Pedrajas et al. 2010; Kretschmer et al. 2012).

Since ancient times, Mexican farmers have harvested the galls of the infected ears to prepare a popular, traditional dish named 'huitlacoche' (Allen et al. 2011; Dean et al. 2012). Because in the USA and some countries of the EU can be considered this high-protein fungus in the preparation of exotic, gourmet dishes in up-market restaurants (Tracy et al. 2007), the commercial potential of developing crop systems enabling production of large quantities of $U$. maydis galls is increasingly being recognised.

Intensive artificial production of $U$. maydis requires a high level of compatibility between the fungus and the host, ideally matching a virulent fungal strain with a highly-susceptible host variety in favourable environmental conditions (Banuett 1995). In order to obtain a large number of galls in the field, conventional method of artificial inoculation by injection of the ear with an inoculum containing a mixture of teliospores (a conservative diploid structure of basidiomycetes) is frequently used. These are germinated on potato dextrose agar (PDA) medium to form haploid sporidia, which are placed in a flask and diluted to $10^{6}$ sporidia $\mathrm{mL}^{-1}$. Optimally, the galls are collected from several regions to increase the probability of including sporidia of a range of mating types to create a highlyinfective inoculum (Snetselaars \& Mims 1993).

However, in field experiments this method produces erratic results in terms of the incidence and severity of fungal infection. Results are even more variable when this technique is applied on different maize genotypes and or in a range of environments. Indeed, results are quite variable even under optimised greenhouse conditions with disease incidence and severity varying from 1 to $53 \%$ (Madrigal-Rodríguez et al. 2010). To obtain an infective inoculum capable of producing consistent gall formation in a range of maize cultivars, it is necessary to understand the biology and life cycle of the disease. We know that $U$. maydis forms three cell types during its life cycle: (i), haploid, unicellular, yeast-like sporidia, (ii) dikaryotic, filamentous hyphal cells resulting from crosses between two different haploid mycelia, and (iii) diploid teliospores (Schulz et al. 1990). However, it is important to consider that haploid cells are saprotrophic and nonpathogenic, in contrast to the dikaryotic forms that are pathogenic. The latter are not cultivable in artificial media but it is the most frequent form found in infected host plants. Considering its heterothallic characteristic, mating between haploid cells is controlled by two genetic loci, the $a$ locus with two alleles controls cell fusion, and the $b$ locus which is multiallelic with at least 25 alleles (Kahmann \& Kämper 2004). The last controls events after fusion including fungus development within the maize plant. Each combination of $b$ alleles can be distinguished by a homozygous or heterozygous stage, which influences the pathogenicity, sexual development and cell morphology (Romeis et al. 2000). The $b$ locus presents an excellent opportunity for studying the mechanism of self-versus non-self-recognition involving a multiallelic gene in a relatively simple organism (Kronstad \& Leong 1989). Also, in the absence of pathogenic mating, yeast-like colonies can give rise to diploid 'solopathogenic' hyphae (Banuett \& Herskowitz 1989). It has been estimated that approximately $2.6 \%$ of sporidia possess this feature (Sabbagh et al. 2010). Based on this, a way to produce virulent inoculum is via solopathogenic hyphae isolated from yeast-like sporidia or from dikaryotic mycelia formed by mating two haploid hyphae. In nature, a 10-cm long gall contains about 200 trillion of teliospores (Madrigal-Rodríguez et al. 2010), each with the capacity to produce cells of a unique genotype, offer the opportunity to isolate pathogenic strains in vitro using the fuzz reaction (Banuett \& Herskowitz 1994; Chew et al. 2008).

The aim of this study was to determine whether it is possible to isolate teliospores from maize ears infected with U. maydis under field conditions in Mexico, with compatible sporidia or solopathogenic strains able to produce a highly infective inoculum. For this, optical microscopy was used alongside partial-diallel type arrangements of paired strains and phylogenetic identification methods using PCR amplification of the $b$ locus in $U$. maydis strains obtained from galls, recovered from landrace maize fields adapted to the Mexican Highlands.

\section{Materials and methods}

\section{Experimentalmaterial}

Twenty ears with gall symptoms were collected during the spring-summer growing season in 2010-2011, from experimental maize plots located at Montecillo, State of México, in the Mexican Highlands (2250 m, altitude). The galls came from two open-pollinated experimental maize varieties, Montecillo and Santos. Maize kernels containing teliospores were separated from the ears (Fig. 1).

\section{Isolation of teliospores}

From each gall, $10 \mathrm{mg}$ of teliospore material was weighed and placed in an eppendorf tube containing $1 \mathrm{~mL}$ of sterile distilled water and $2 \mu \mathrm{L}$ of Tween 20 . The tubes were shaken gently until the teliospores were observed to be well dispersed in the solution. Each tube was serially diluted from $10^{-1}$ to $10^{-10}$, and each dilution step was plated in triplicate on Petri plates containing PDA medium (Bioxon, USA).

After $48 \mathrm{~h}$ incubation at room temperature $\left(\right.$ c. $20^{\circ} \mathrm{C}$ ) yeast-like colonies were observed. A total of 26 colonies were selected on the criterion that each isolate was the result of 


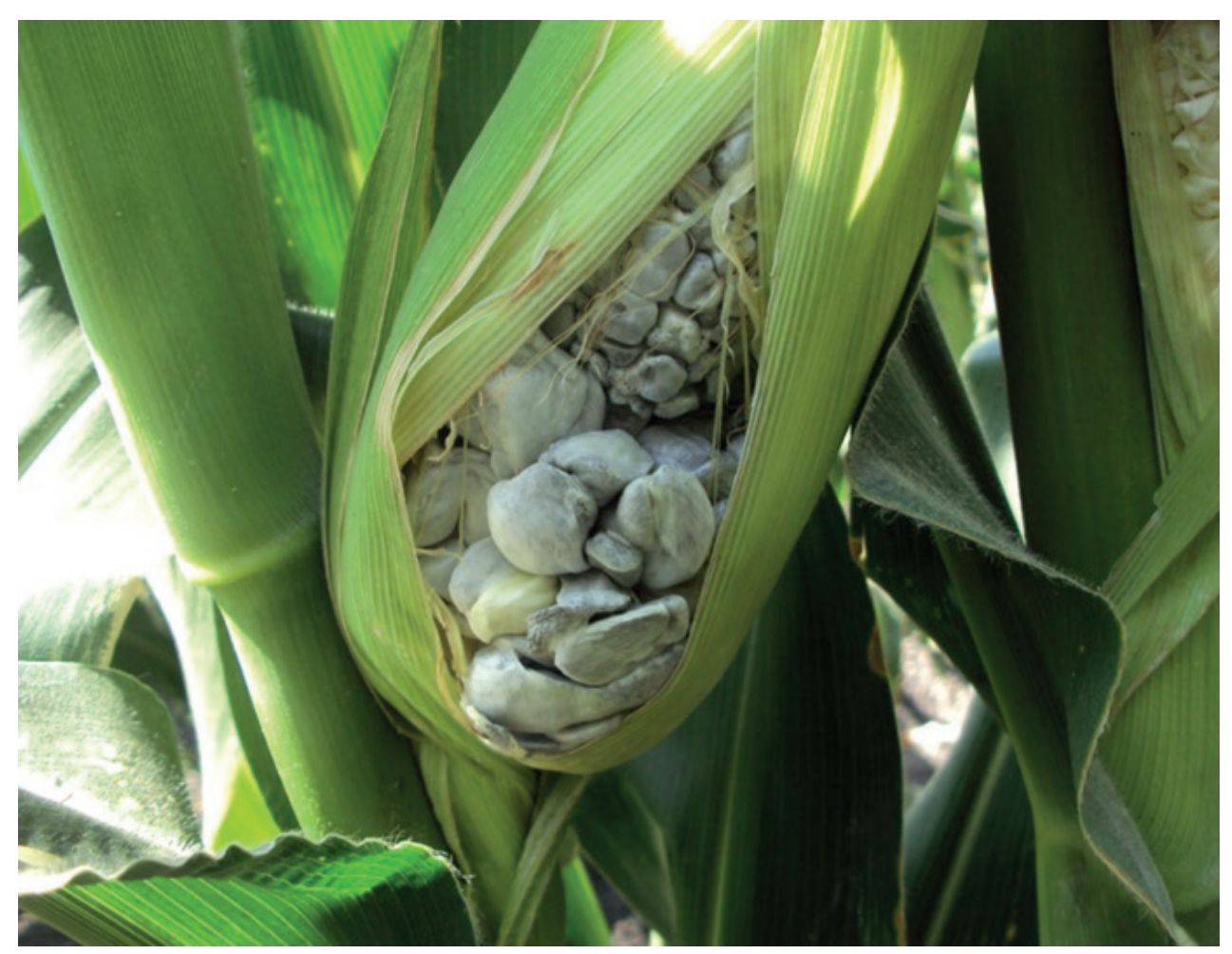

Figure 1. Maize ears showing gall symptoms in Montecillo-Mexico during the spring-summer growing season 2010-2011. These galls came from 'Santos', an open-pollinated experimental maize variety.

a teliospore germination. These were identified as 1 to 15 (from Montecillo) and 16 to 26 (from Santos).

\section{Detection of pathogenic strains}

Colonies developed on PDA medium supplemented with $5 \%$ activated charcoal powder (PDA+AC) (Merck, Germany) were assessed individually and their growth features were scored for eight days according MartínezEspinoza et al. (1997).

An initial evaluation was made after incubation at room temperature for $48 \mathrm{~h}$. All strains were classified according to their growth as: (-) a yeast-like colony of rough or smooth appearance but without a surrounding mycelium, $(+)$ a yeastlike colony with sparsely-developed mycelium around the colony, and $(++)$ a yeast-like colony surrounded by a dense, cottony mycelium. Strains were considered pathogenic when sparse or cottony mycelia grew around the colony.

\section{Selection of solopathogenic strains}

All strains showing a growth of sparse or cottony mycelium were transferred by four or five successive streaks to the PDA+AC in which pathogenic strains were selected. In this study, strains were considered solopathogenic when the cottony mycelia were unaltered after successive subcultures (Sabbagh et al. 2010).

\section{Partial-diallel arrangement of strains}

The 26 strains were plated in pairs on Petri plates containing PDA+AC medium resembling a partial-diallel arrangement (Rowell \& DeVay 1954) (Tab. 1), so that mating type compatibility could be observed by the growth mycelium. This positive phenotype is termed fuzz reaction (Martínez-Espinoza et al. 1997).

In this test, dikaryotic mycelium formed in vitro by mating between two compatible haploid hyphae, may give rise to white colonies with sparse or cottony appearances. Strains that do not mate directly develop grayish, yeast-like colonies indicating incompatibility between hyphae grown in the same medium (Brefort et al. 2009).

\section{DAPl staining}

To confirm the presence of diploid sporidia and clampconnection in solopathogenic strains, $1 \mathrm{~mL}$ of sterile distilled water was added to each plate to detach the sparse mycelium. To stain the DNA, a drop of this suspension was placed on a slide with $0.5 \mu \mathrm{g} \mathrm{mL}^{-1}$ of the reagent 4', 6-diamino2-phenylindole (DAPI) in phosphate-buffered saline $(\mathrm{pH}$ 7.2) for $20 \mathrm{~min}$ at $60{ }^{\circ} \mathrm{C}$ and subsequently washed with the same buffer (Dann et al. 1971). After $20 \mathrm{~min}$, samples 
Porfirio Raúl Galicia-Garcia, Hilda Victoria Silva-Rojas, Leopoldo Ernesto Mendoza-Onofre,

Hilda Araceli Zavaleta-Mancera, Leobigildo Córdova-Téllez and Alejandro Espinosa-Calderón

Table 1. Morphology of 26 colonies from Ustilago maydis in partial-diallelic arrangement evaluated on PDA medium supplemented with a $5 \%$ carbon source.

\begin{tabular}{|c|c|c|c|c|c|c|c|c|c|c|c|c|c|c|c|c|c|c|c|c|c|c|c|c|c|c|}
\hline $\begin{array}{l}\text { CPO } \\
\text { strain }\end{array}$ & 1 & 2 & 3 & 4 & 5 & 6 & 7 & 8 & 9 & 10 & 11 & 12 & 13 & 14 & 15 & 16 & 17 & 18 & 19 & 20 & 21 & 22 & 23 & 24 & 25 & 26 \\
\hline 1 & & ++ & ++ & +- & ++ & +- & +- & ++ & ++ & +- & ++ & ++ & ++ & ++ & +- & +- & ++ & ++ & ++ & +- & ++ & ++ & ++ & ++ & ++ & ++ \\
\hline 2 & & & ++ & +- & ++ & +- & +- & ++ & ++ & +- & ++ & ++ & ++ & ++ & +- & +- & ++ & ++ & ++ & +- & ++ & ++ & ++ & ++ & ++ & ++ \\
\hline 3 & & & & +- & ++ & +- & +- & ++ & ++ & +- & ++ & ++ & ++ & ++ & +- & +- & ++ & ++ & ++ & +- & ++ & ++ & ++ & ++ & ++ & ++ \\
\hline 4 & & & & & -+ & -- & -- & -+ & -+ & -- & -+ & -+ & -+ & -+ & -- & -- & -+ & -+ & -+ & -- & -+ & -+ & -+ & -+ & -+ & -+ \\
\hline 5 & & & & & & +- & +- & ++ & ++ & +- & ++ & ++ & ++ & ++ & +- & +- & ++ & ++ & ++ & +- & ++ & ++ & ++ & ++ & ++ & ++ \\
\hline 6 & & & & & & & -- & -+ & -+ & -- & -+ & -+ & -+ & -+ & -- & -- & -+ & -+ & -+ & -- & -+ & -+ & -+ & -+ & -+ & -+ \\
\hline 7 & & & & & & & & -+ & -+ & -- & -+ & -+ & -+ & -+ & -- & +- & -+ & -+ & -+ & -- & -+ & -+ & -+ & -+ & -+ & -+ \\
\hline 8 & & & & & & & & & ++ & +- & ++ & ++ & ++ & ++ & +- & +- & ++ & ++ & ++ & +- & ++ & ++ & ++ & ++ & ++ & ++ \\
\hline 9 & & & & & & & & & & +- & ++ & ++ & ++ & ++ & +- & +- & ++ & ++ & ++ & +- & ++ & ++ & ++ & ++ & ++ & ++ \\
\hline 10 & & & & & & & & & & & -- & -+ & -+ & -+ & -- & -- & -+ & -+ & -+ & -- & -+ & -+ & -+ & -+ & -+ & -+ \\
\hline 11 & & & & & & & & & & & & ++ & ++ & ++ & +- & +- & ++ & ++ & ++ & +- & ++ & ++ & ++ & ++ & ++ & ++ \\
\hline 12 & & & & & & & & & & & & & ++ & ++ & +- & +- & ++ & ++ & ++ & +- & ++ & ++ & ++ & ++ & ++ & ++ \\
\hline 13 & & & & & & & & & & & & & & ++ & +- & +- & ++ & ++ & ++ & +- & ++ & ++ & ++ & ++ & ++ & ++ \\
\hline 14 & & & & & & & & & & & & & & & +- & +- & ++ & ++ & ++ & +- & ++ & ++ & ++ & ++ & ++ & ++ \\
\hline 15 & & & & & & & & & & & & & & & & -- & -+ & -+ & -+ & -- & -+ & -+ & -+ & -+ & -+ & -+ \\
\hline 16 & & & & & & & & & & & & & & & & & -+ & -+ & -+ & -- & -+ & -+ & -+ & -+ & -+ & -+ \\
\hline 17 & & & & & & & & & & & & & & & & & & ++ & ++ & +- & ++ & ++ & ++ & ++ & ++ & ++ \\
\hline 18 & & & & & & & & & & & & & & & & & & & ++ & +- & ++ & ++ & ++ & ++ & ++ & ++ \\
\hline 19 & & & & & & & & & & & & & & & & & & & & +- & ++ & ++ & ++ & ++ & ++ & ++ \\
\hline 20 & & & & & & & & & & & & & & & & & & & & & -+ & -+ & -+ & -+ & -+ & -+ \\
\hline 21 & & & & & & & & & & & & & & & & & & & & & & ++ & ++ & ++ & ++ & ++ \\
\hline 22 & & & & & & & & & & & & & & & & & & & & & & & ++ & ++ & ++ & ++ \\
\hline 23 & & & & & & & & & & & & & & & & & & & & & & & & ++ & ++ & ++ \\
\hline 24 & & & & & & & & & & & & & & & & & & & & & & & & & ++ & ++ \\
\hline 25 & & & & & & & & & & & & & & & & & & & & & & & & & & ++ \\
\hline
\end{tabular}

+presence of mycelia; - absence of a surrounding mycelium; ${ }^{++}$compatible mating type. Solopathogenic strains (sign in bold).

were observed under a fluorescence microscope with mercury short arc lamps $\mathrm{HBO} 50 \mathrm{~W}$ at a wavelength of 350 nanometers (Axiolab, Carl Zeiss, Germany). The infective potential of each solopathogenic strain was corroborated in maize seedlings in a greenhouse and in the field studies (unpubl.res.).

\section{DNA extraction}

Total DNA was extracted from saprotrophic, pathogenic and solopathogenic representative strains by addition of $1 \mathrm{~mL}$ of CTAB $2 \%$ (Doyle \& Doyle 1990). Subsequently, $600 \mu \mathrm{L}$ of the solution was recovered from the Petri plate and transferred to a $2 \mu \mathrm{L}$ eppendorf tube to continue DNA extraction, including a washing with $3 \mathrm{M}$ sodium acetate. The DNA pellet from each tube was re-suspended in 50 $\mu \mathrm{L}$ of TE buffer (10 mM Tris- $\mathrm{HCl}, \mathrm{pH} 8,1 \mathrm{mM}$ EDTA). The quality of the DNA was verified by electrophoresis in a $1.5 \%$ agarose gel in $1 x$ TAE buffer (Tris Acetate EDTA) run at $87 \mathrm{~V} \mathrm{~cm}^{-1}$ for $1 \mathrm{~h}$. The gel was stained with $\mathrm{Gel}$ Red (Biotium, Ca, USA), and the bands were visualised using an Infinity 1000/26MXXpress (Vilber Lourmat, Germany) gel documentation system. The DNA concentration was quantified using a Nanodrop $2000 \mathrm{C}$ spectrophotometer Lambda BIO 10 (Thermo Scientific, USA), and the samples were diluted to $20 \mathrm{ng} \mu \mathrm{L}^{-1}$ for amplification of the $b$ alleles.

\section{PCR amplification and sequencing}

Specific primers $b E 4$ (5'-CTCGAGGTTCATCAGCTCA-3') and $b E 8$ (5'-GCTGAGTTCTGGAGTCG-3') to amplify $b \mathrm{E}$ locus to determinate the type of regulatory protein were used (Albert \& Schenck 1996). A PCR master mix was prepared in a final volume of $25 \mu \mathrm{L}$ containing $1 x$ taq DNA polymerase buffer, $0.8 \mu \mathrm{M}$ deoxynucleotides ( $0.2 \mathrm{mM}$ each), 100 ng DNA, 10 pmol of each primer, and 2 Units of Taq DNA polymerase (Promega, USA). 
PCR amplifications were performed with an initial denaturation at $95^{\circ} \mathrm{C}$ for $2 \mathrm{~min} ; 35$ cycles of denaturation at $95^{\circ} \mathrm{C}$ for $1 \mathrm{~min}$, annealing at $55^{\circ} \mathrm{C}$ for $1 \mathrm{~min}$, and extension at $72{ }^{\circ} \mathrm{C}$ for $2 \mathrm{~min}$; followed by a final extension at $72{ }^{\circ} \mathrm{C}$ for $10 \mathrm{~min}$. PCR reactions were carried out in a Peltier Thermal Cycler PTC-200 (Bio-Rad, USA), and the PCR products were verified by loading $5 \mu \mathrm{L}$ in a $1.5 \%$ agarose electrophoresis gel, which was stained as described above. The remaining amplified $P C R$ products were purified using ExoSAP-IT (Affymetrix, Santa Clara CA, USA) following the manufacturer's instructions.

Sequencing reactions were prepared with big dye terminator v3.0 (Applied BioSystems, Ca, USA), and fragments were resolved in an automated Genetic Analyser model 3730 DNA (Applied BioSystems, Ca, USA). To avoid misreadings, the PCR products were sequenced in both directions. Sequences corresponding to regulatory protein from new $U$. maydis isolates were deposit in GenBank from the National Center for Biotechnology Information (NCBI) under accession number KF647221-KF647225 and KF689679-KF689685. For cases in which multiple isolates had identical sequences, only one accession number per sequence was deposited, representing the common sequence of those isolates.

\section{Analysis of b-locus diversity}

Sequences corresponding to both strands of the $b \mathrm{E}$ gene were assembled and edited using BIOEDIT v7.2.0 software (Hall 1999), and a consensus sequence of each isolate was created and compared with GenBank from NCBI database through BLASTN 2.2.19 option (Zhang et al. 2000).

For evolutionary analysis, two different methods were performed. In the first one, all consensus sequences were compiled into a single file (FASTA format) to build a nucleotide sequence alignment with the profile mode of ClustalW 1.8.1 (Thompson et al. 1994). Phylogenetic reconstruction was performed with $b$ E sequences using the statistical method of Maximum Parsimony (MP) included in the MEGA 6 software (Tamura et al. 2013). To determine the confidence values for clades within the resulting tree, a bootstrap analysis was assessed with 1000 replicates (Felsenstein 1985). Data subset was conducted using the close-neighbour interchange (CNI) on random trees, with MP search option (level =1) and initial tree by random addition (10 reps), and gapsimissing data were considered a complete deletion. The accession numbers of $U$. maydis deposited in the GenBank - NCBI database corresponding to $b 1, b 2, b 3$ and $b 4$, are different allele of the same gene, they were downloaded and included as reference sequences along with the sequences obtained in this study. Sporisorium reilianum (accession number EU167575) was designated as out group.

In the second one, Bayesian inference was calculated using MrBayes v3.2.2 (Ronquist \& Huelsenbeck 2003) with
Markov Chain Monte Carlo (MCMC) algorithm to generate trees with Bayesian posterior probabilities. Three heated chains and one cold chain with 200,000 generations were run to reach a $\mathrm{P}$ value lower than 0.01 with two parallel searches considering a sample frequency of 1 in 100. To obtain the consensus tree, we used the most complex model of evolution available for nucleotides (GTR + gamma distribution + invariant positions). The first $25 \%$ of trees were discarded as the burn-in phase of each analysis and posterior probabilities was determined for the remaining trees. The consensus tree was visualized with FigTree v1.4 (Rambaut \& Drummond 2010) and export to Mega 6 software for final print.

\section{Results}

\section{Teliospore germination}

A mass of sooty appearance of melanized teliospores was collected from inside galls produced from infected maize kernels. Diploid teliospores placed on PDA medium germinated after three days, and yeast-like colonies were observed. Galls of $U$. maydis collected from maize fields are associated with a complex of yeast-like colonies of similar colour and consistency, among these are: Candida railenensis, C. quercitrusa and Pichia guilliermondii. For this reason phylogenetic identification was performed with all strains obtained from teliospores germinated in vitro, to be sure that analysis was carried out with $U$. maydis strains.

\section{Microscopic observation}

When yeast type colonies cultured on PDA were transferred in PDA+AC medium, three types of colonies development were observed after $72 \mathrm{~h}$. The first colony type was formed by saprotrophic strains with yeast-like colonies with no mycelium. These were bright or opaque in appearance, cream or pale yellow in colour and had either a smooth or rough surface (Fig. 2A). The second colony type was characterised as pathogenic strains because their yeastlike colonies were white and had rough surfaces with sparse mycelium (Fig. 2B). In the third colony type, the pathogenic, yeast-like colonies developed a cottony mycelium. These colonies were initially white but became brown after $72 \mathrm{~h}$. All strains of this type retained the same morphology after five subcultures (Fig. 2C).

\section{Detection of compatibility group}

The partial-diallel strain arrangement allowed determination in vitro of compatible and incompatible matings, as well as saprotrophic and pathogenic combination (Fig. 3). The growth of mycelial fuzz was evident only when sexually compatible hyphae fused (Tab. 1). 
Porfirio Raúl Galicia-García, Hilda Victoria Silva-Rojas, Leopoldo Ernesto Mendoza-Onofre, Hilda Araceli Zavaleta-Mancera, Leobigildo Córdova-Téllez and Alejandro Espinosa-Calderón
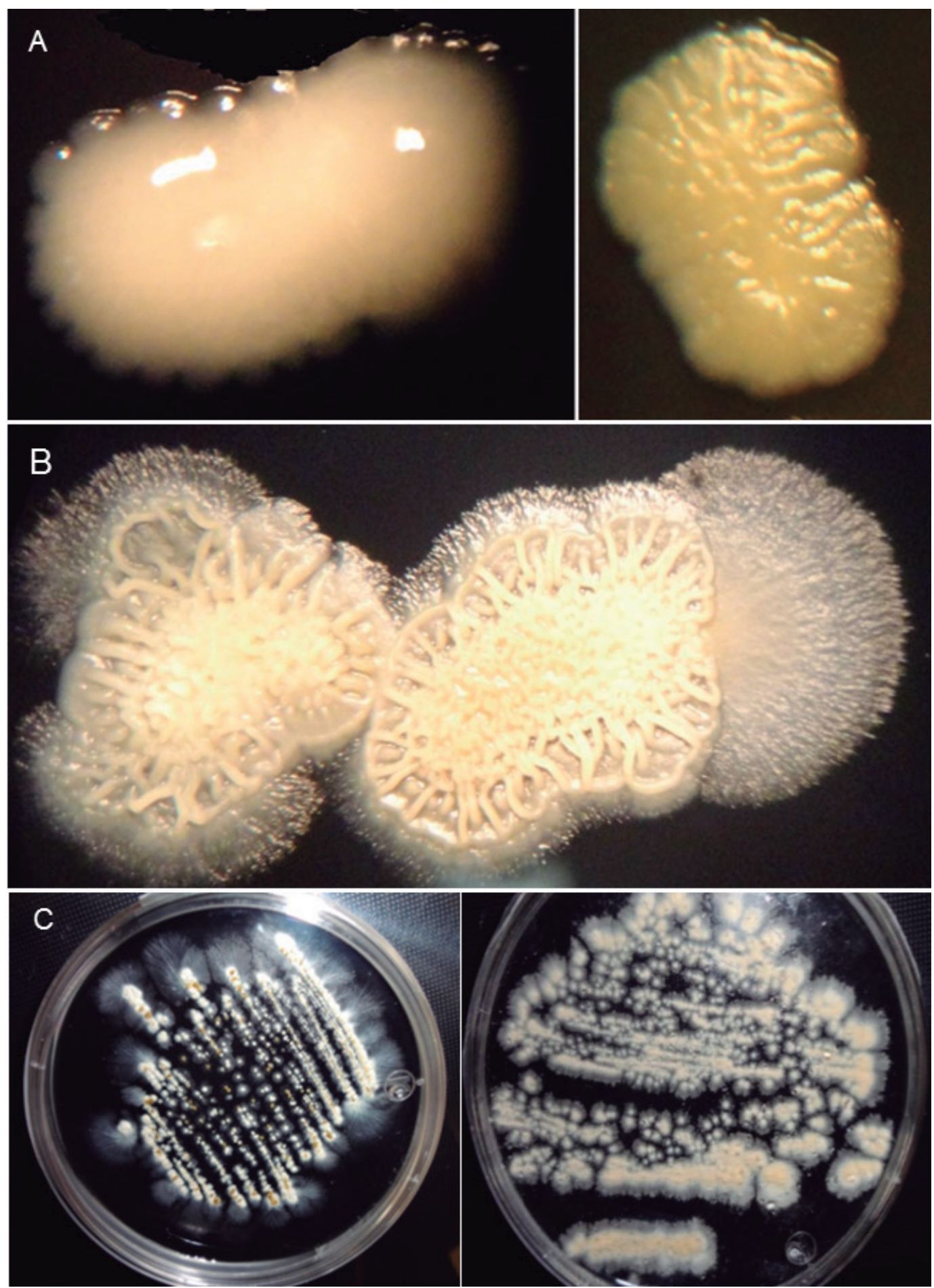

Figure 2. Yeast-like colonies of Ustilago maydis collected from landrace maize in the Mexican Highlands, grown on a PDA medium supplemented with a 5\% carbon source. Panel A: yeast-like colony with no mycelium, bright or opaque in appearance, cream or paleyellow in colour and having a rough or smooth surface. Panel B: yeast-like colony, white and with a rough surface, and an obvious surrounding filamentous mycelium. Panel C: yeast-like, white colony developing cottony mycelium but becoming brown after $72 \mathrm{~h}$, that retained their properties after five re-streaks under in vitro conditions.

The group of 26 strains in 325 pairs of combinations were characterised individually (Tab. 1). When grown on a $\mathrm{PDA}+\mathrm{AC}$, all showed a yeast-like colony with a smooth or rough surface. When the strains were placed in pairs, most produced rough-textured colonies with filamentous mycelia at the periphery. In Table 1, '+' indicates when a peripheral mycelium was present and '-' when absent.

Analysis of the mating behaviour of the 26 colonies 


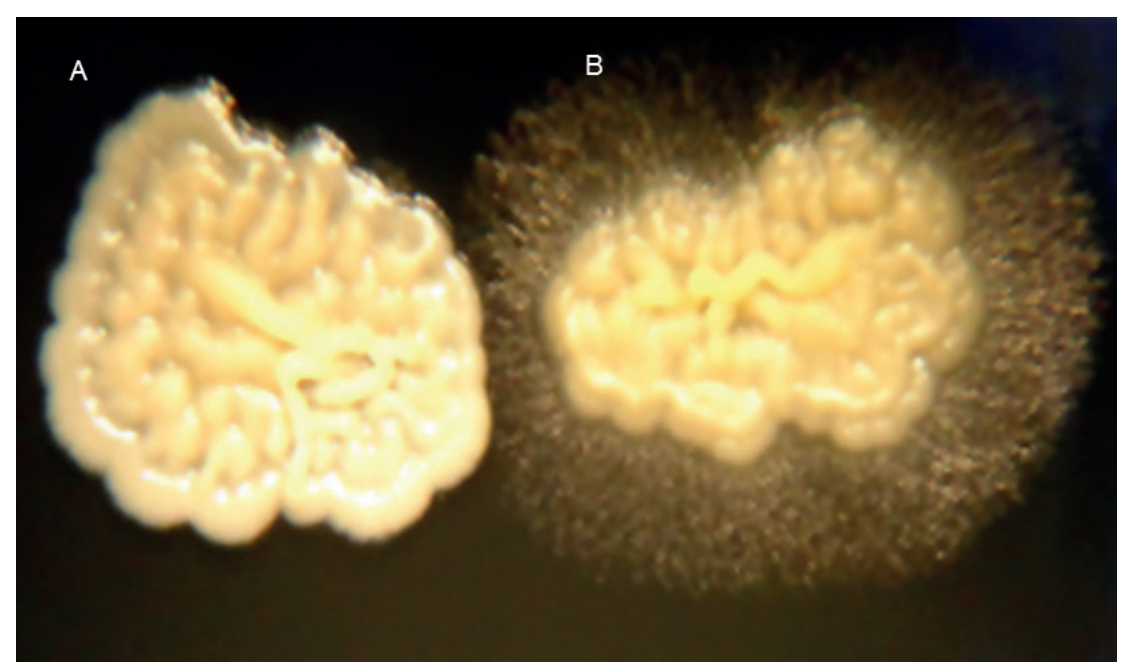

Figure 3. Colonies of Ustilago maydis developed on PDA medium supplemented with a 5\% carbon source. A: Saprotrophic colony showed a rough surface. B: Colony with a rough surface developing a peripheral mycelium.

(Tab. 2) indicates that CPO- 1 strain in combinations with others resulted in the detection of four solopathogenic strains; they showed rough colonies and peripheral mycelia in all cells. In two strains (CPO-5 and $\mathrm{CPO}-8)$ there were three; in five strains (CPO-2, CPO-3, CPO-9, CPO-11 and $\mathrm{CPO}-18)$ there was only one. Growth showed differences in the size of colonies, as well as repeated streaks of each colony. Fifteen strains were identified as solopathogenic (signed in bold, Tab. 1).

\section{DAPl staining}

Sporidia of Ustilago maydis having one nucleus was visualised by DAPI staining (Fig. 4). On the other hand, the dikaryotic growth stage was also evident in Petri plates, plated under laboratory conditions in pathogenic strains. Solopathogenic diploid sporidia are not a product of the fusion of two compatible haploid hyphae but are formed during teliospore germination. The nucleus migrates to the developing pro-mycelium centre, where the first meiotic division occurs. Usually, but not always, four cells remains in the pro-mycelium, and each gives rise to a single primary sporidium. While this description of the germination and the formation of sporidia is classic, it is not the most common process. A single pro-mycelium can lead to five or seven primary sporidia and some of these can be diploid (Sabbagh et al. 2010). These diploid sporidia give rise to solopathogenic strains.

\section{Amplification of the b-locus diversity}

PCR amplification with primers to identify a partial sequence of the $b \mathrm{E}$ locus of $U$. maydis was successful. A 480 bp fragment was clearly observed by agarose electrophoresis gel.
Twelve representative sequences corresponding to amplification of the partial $b$ locus were used to establish the type of regulatory protein and phylogenetic reconstruction. The resulting consensus bootstrap tree performed with maximum parsimony method and Bayesian inference show that the strains clearly fell into two clades. In maximum parsimony method Clade I: the strains CPO-5af, CPO-5bf, CPO-15af, CPO-15bf, CPO-20af, CPO-20bf, CPO23af, CPO-23bf, CPO-24af and CPO-24bf were grouped with GenBank accession number M58556, belonging to $U$. maydis b4 protein. In Clade II, the strains CPO-9af and CPO-9bf were grouped with GenBank accession number M58555 belonging to U. maydis $b 3$ protein. Both accessions corresponded to sequences obtained from RK32 and RK138 respectively selected as meiotic segregants from $U$. maydis teliospores collected in the Bonn area in Germany (Schulz et al. 1990). No single sequences matched with accession number M58553 U. maydis $b 1$ protein or with M58554 $U$. maydis $b 2$ protein (Fig. 5). In the same way, Bayesian inference grouped all the strains into two clades showing consistency in both tree, $p$ value after 200,000 generations was 0.008 .

\section{Discussion}

Although U. maydis is widely studied, few works deal on the diversity of Mexican or Mesoamerican strains. We here report an original work to isolate aggressive solopathogenic strains suitable for artificial inoculation in this part of the world where maize is the main crop. In this research, we present the results of attempts to isolate teliospores collected from galls formed in ears of field-grown, landrace maize adapted to the Mexican Highlands, and their ability to form the dikaryotic filamentous mycelia required to initiate the pathogenic process, and the existence of solopathogenic strains. 
Porfirio Raúl Galicia-García, Hilda Victoria Silva-Rojas, Leopoldo Ernesto Mendoza-Onofre, Hilda Araceli Zavaleta-Mancera, Leobigildo Córdova-Téllez and Alejandro Espinosa-Calderón

Table 2. Light microscope study of the morphologies of 26 Ustilago maydis strains and their partial-diallelic arrangements evaluated on PDA medium supplemented with a $5 \%$ carbon source.

\begin{tabular}{|c|c|c|c|}
\hline \multirow{2}{*}{$\begin{array}{l}\text { CPO } \\
\text { Strain }\end{array}$} & \multicolumn{2}{|c|}{ Yeast-like colony ${ }^{a}$} & \multirow{2}{*}{$\begin{array}{l}\text { Number of mating pairs with solopathogenic } \\
\text { strain }\end{array}$} \\
\hline & $\begin{array}{c}\text { Smooth surface without mycelia } \\
\text { [-] }\end{array}$ & $\begin{array}{l}\text { Rough surface and weak peripheral mycelium } \\
{\left[\begin{array}{l}+ \\
\text { nom }\end{array}\right.}\end{array}$ & \\
\hline 1 & 0 & 25 & [1x9], [1x11], [1x12],[1x23] \\
\hline 2 & 0 & 25 & {$[2 \times 22]$} \\
\hline 3 & 0 & 25 & {$[3 \times 14]$} \\
\hline 4 & 25 & 0 & 0 \\
\hline 5 & 0 & 25 & [5x9], [5x14], [5x19] \\
\hline 6 & 25 & 0 & 0 \\
\hline 7 & 25 & 0 & 0 \\
\hline 8 & 0 & 25 & [8x9], [8x13],[8x17] \\
\hline 9 & 0 & 25 & {$[9 \times 17]$} \\
\hline 10 & 25 & 0 & 0 \\
\hline 11 & 0 & 25 & {$[11 \times 18]$} \\
\hline 12 & 0 & 25 & 0 \\
\hline 13 & 0 & 25 & 0 \\
\hline 14 & 0 & 25 & 0 \\
\hline 15 & 25 & 0 & 0 \\
\hline 16 & 25 & 0 & 0 \\
\hline 17 & 0 & 25 & 0 \\
\hline 18 & 0 & 25 & [18x26] \\
\hline 19 & 0 & 25 & 0 \\
\hline 20 & 25 & 0 & 0 \\
\hline 21 & 0 & 25 & 0 \\
\hline 22 & 0 & 25 & 0 \\
\hline 23 & 0 & 25 & 0 \\
\hline 24 & 0 & 25 & 0 \\
\hline 25 & 0 & 25 & 0 \\
\hline 26 & 0 & 25 & 0 \\
\hline
\end{tabular}

aTwenty-six strains of Ustilago maydis evaluated in 25 partial-diallelic combinations.

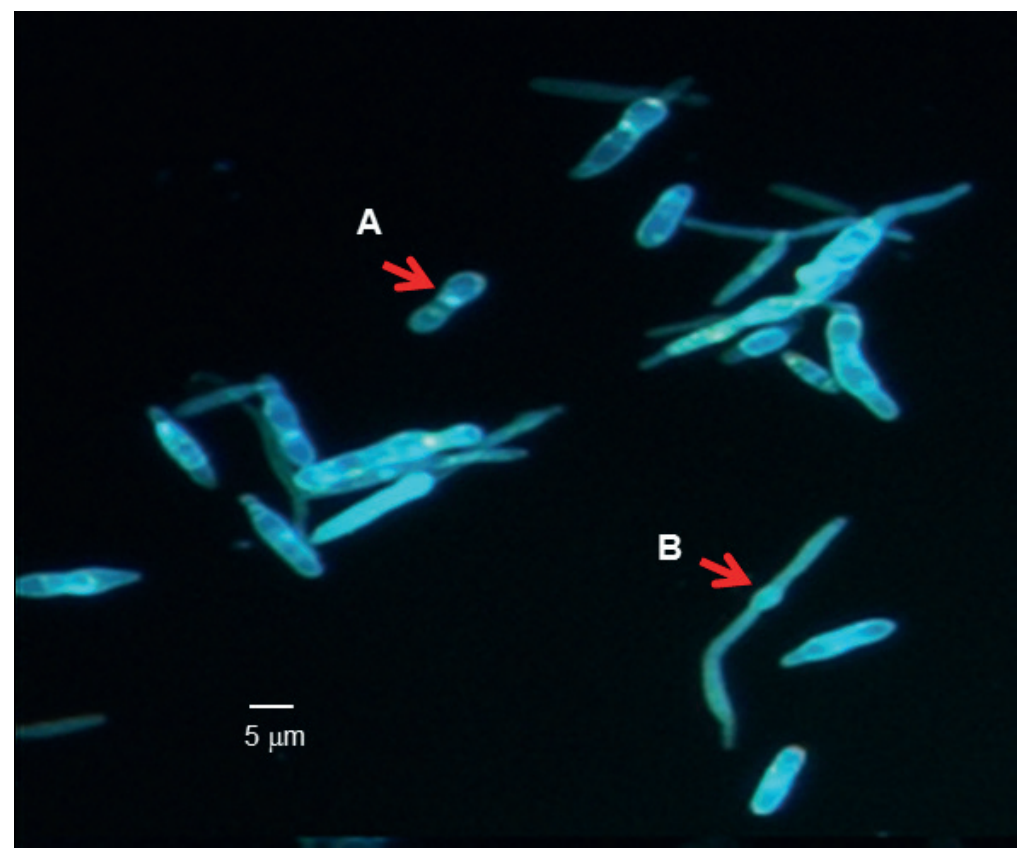

Figure 4. DAPI staining of Ustilago maydis $\mathrm{CPO}-23$ solopathogenic strain observed with fluorescence microscopy. A: diploid sporidia and B: clamp-connection structure. Scale bar. $5 \mathrm{~mm}$ 


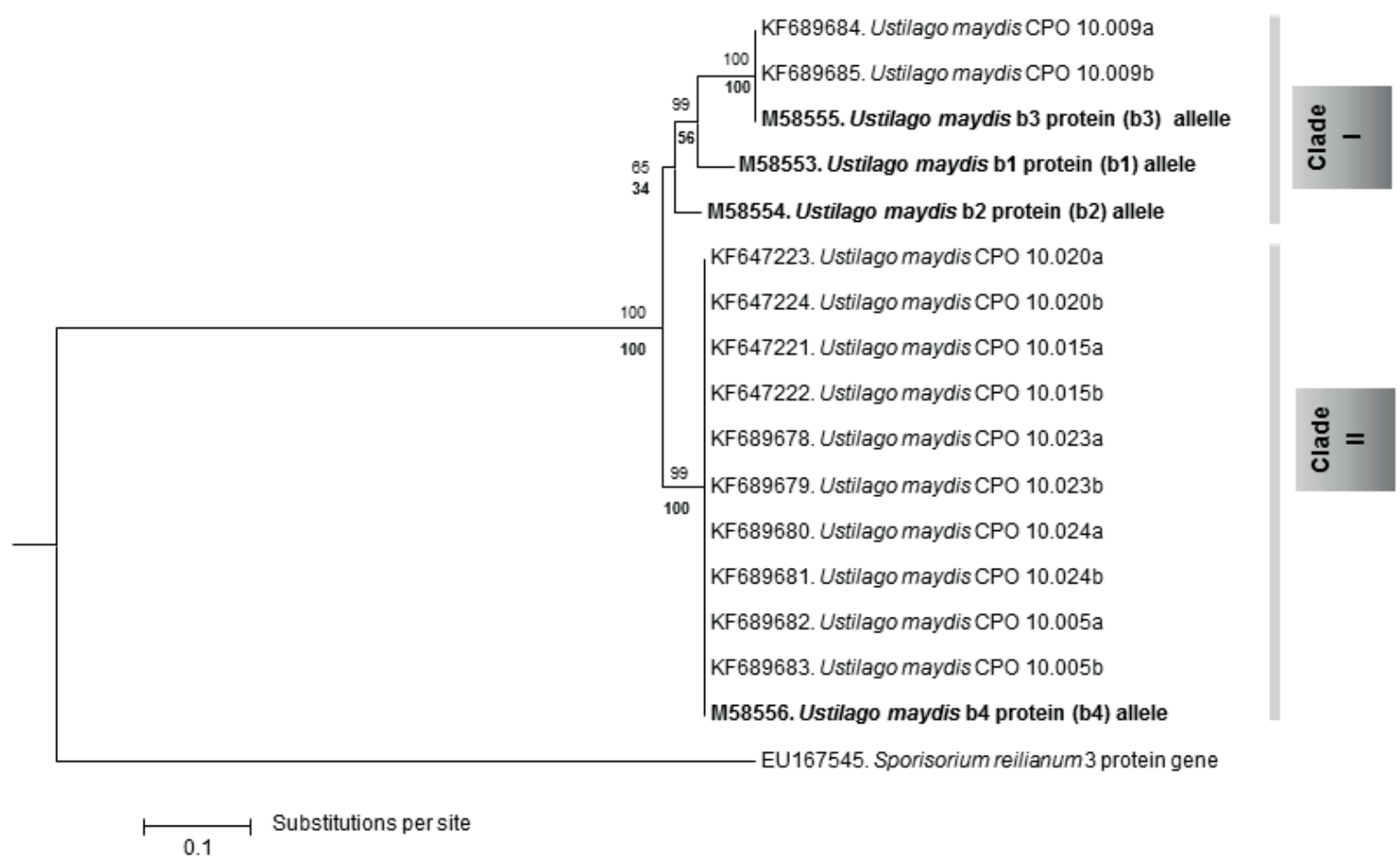

Figure 5. Phylogenetic consensus tree based on Bayesian inference, showing the relationship between Ustilago maydis sequences of partial $b$ locus obtained of teliospores isolated from galls in Mexican Highland field and the four alleles reported previously of the same locus. The bootstrap confidence value is displayed in each cluster and for maximum parsimony method is shown in bold.

Yeast-like colonies obtained by germinating teliospores, were grown on an artificial medium supplemented with a carbon source. These colonies have different forms and various colours. In some strains, there was a peripheral growth of filamentous mycelium, in line with studies on fungal morphology, physiology and ultrastructure performed in other parts of the world where maize is grown (Kahmann \& Kämper 2004). As known for U. maydis, we obtained three types of stages: i) diploid teliospores, conservative pathogenic structures that form inside galls, germinate outside the host to produce septate basidia on which haploid basidiospores (sporidia) are borne, ii) saprotrophic mycelia arise from germinated haploid unicellular sporidia that divide by budding, to form yeast-like colonies, and iii) filamentous dikaryotic hyphae, formed as the product of sexual reproduction between compatible haploid cells colonise the host plant without inducing defence responses (Mueller et al. 2013) and induce the formation of galls (sori). While formation and development of the dikaryon is restricted to maize tissue, the haploid mycelia and diploid mycelia from solopathogenic strains can grow well on artificial media.

Meanwhile, the $b$ locus regulates the combination of any two different alleles that triggers the pathogenicity response, but not when the alleles are identical. Resulting from these combinations, it can be observed the development of yeast-like colonies taking one of three forms: a colony with no surrounding mycelium, a colony with a sparse surrounding mycelium or a colony surrounded by a dense, cottony mycelium in the case of solopathogenic strains (Fig. $2 \mathrm{C}$ ). Light microscope studies of the morphology of these colonies revealed the presence in the mycelia of specialised structures known as clamp connections. These are one of the most characteristic features of basidiomycetes. DAPI staining confirmed the presence of haploid sporidia that give rise to solopathogenic strains that form stable colonies through successive re-streaks on artificial media. Later, amplification and sequencing by chain termination (Sanger sequencing) of the partial $b$ E gene were carried out because phylogenetic identification of the Ustilaginales using largesubunit, nuclear ribosomal DNA sequences does provide ambiguous identification either among or within species (Stoll et al. 2003). Our results obtained using $b$ E sequencing were particularly interesting because they revealed only the presence of alleles that encode $b 3$ protein (B3) and $b 4$ protein (B4) (Fig. 5). These alleles were first reported by Schulz et al. (1990), as functionally distinct from $b 1, b 2$, and each other protein regulator that encodes for polypeptides containing a homeodomain-related motif.

We infer that strains collected from U. maydis galls in the Mexican Highlands are closely related to the strains described by Schultz et al. (1990). The combination $a 1 / b 3$ and $a 1 / b 4$ alleles are the most common in this area and 
they are not efficient because a1/a2 is necessary. However, it is necessary to collect galls from other areas of Mexico to determinate (i) whether $\mathrm{a} 1 / \mathrm{b} 1$ or $\mathrm{a} 1 / \mathrm{b} 2$ alleles are present elsewhere in Mexico and (ii) to identify the role of these alleles in the pathogenicity process because both genes are considered to regulatory proteins related with development and pathogenicity.

In summary, our study has identified strains of $U$. maydis that develop yeast-like colonies with mycelia haploid or with solopathogenic properties, thus creating the underlying knowledge base required to begin the development of effective commercial systems for the production of $U$. maydis galls worldwide.

\section{Acknowledgments}

The first author wishes to thank Mexico's National Council of Science and Technology (CONACYT) for the financial support to carry on his studies at the Colegio de Postgraduados. Also, the Seed Pathology and Biotechnology Laboratory at Montecillo Campus and LPI 5 are thanked for making PCR equipment and sequencing facilities available. Lastly, to the Universidad Tecnológica de Tecámac through their PROMEP program, for granting a leave-in absence to obtain a $\mathrm{PhD}$ degree.

\section{References}

Albert HH, Schenck S. 1996. PCR amplification from a homolog of the $b \mathrm{E}$ mating-type gene as a sensitive assay for the presence of the Ustilago scitaminea DNA. Plant Disease 80: 1189-1192.

Allen A, Islamovic E, Kaur J, Gold S, Shah D, Smith TJ. 2011. Transgenic maize plants expressing the Totivirus antifungal protein, KP4, are highly resistant to maize smut. Plant Biotechnology Journal 9: 857864.

Banuett F. 1995. Genetics of Ustilago maydis, a fungal pathogen that induces tumors in maize. Annual Review of Genetics 29: 179-208.

Banuett F, Herskowitz I. 1989. Different $a$ alleles of Ustilago maydis are necessary for maintenance of filamentous growth but not for meiosis. Proceedings of the National Academy of Sciences of the United States of America 86: 5878-5882.

Banuett F, Herskowitz I. 1994. Identification of fuz7, an Ustilago maydis MEK/MAPKK homolog required for a-locus-dependent and independent steps in the fungal life cycle. Genes and Development 8: 1367-1378.

Brefort T, Doehlemann G, Mendoza-Mendoza A, Reissmann S, Djamei A, Kahmann R. 2009. Ustilago maydis as a pathogen. Annual Review of Phytopathology 47: 423-445.

Chew E, Aweiss Y, Lu CY, Banuett F. 2008. Fuz1, a MYND domain protein, protein is required for cell morphogenesis in Ustilago maydis. Mycologia 100: 31-46.

Christensen JJ. 1963. Corn smut caused by Ustilago maydis. American Phytopathological Society Monograph 2: 1-41.

Dann O, Bergen G, Demant E, Volz G. 1971. Trypanocide damidine des 2-phenyl-benzofurans, 2-phenyl-indens und 2-phenyl-indols. Liebigs Annalen der Chemie 749: 68-89.

Dean R, Kan JAL, Pretorius ZA, et al. 2012. The top10 fungal pathogens in molecular plant pathology. Molecular Plant Pathology 13: 414-430.

Djamei A, Kahmann R. 2012. Ustilago maydis: dissecting the molecular interface between pathogen and plant. PLoS Pathog 8(11): e1002955. doi:10.1371/journal.ppat.1002955.
Doyle JJ, Doyle JL. 1990. Isolation of plant DNA from fresh tissue. Focus 12: 13-15.

FAOSTAT. 2013. Statistical yearbook. World food and agriculture. Rome, Food and Agriculture Organisation of the United Nations.

Felsenstein F. 1985. Confidence limits on phylogenies: an approach using the bootstrap. Evolution 39: 783-791.

García-Pedrajas MD, Baeza-Montañez L, Gold SE. 2010. Regulation of Ustilago maydis dimorphism, esporulation, and pathogenic development by a transcription factor with a highly conserved APSES domain. Molecular Plant Microbe Interactions 23: 211-222.

Hall TA. 1999. BioEdit: a user-friendly biological sequence alignment editor and analysis program for Windows 95/98/NT. Nucleic Acids Symposium Series 410: 95-98.

Kahmann R, Kämper J. 2004. Ustilago maydis: how its biology relates to pathogenic development. The New Phytologist 164: 31-42.

Kretschmer M, Klose J, Kronstad JW. 2012. Defects in mitochondrial and peroxisomal $\beta$-oxidation influence virulence in the maize pathogen Ustilago maydis. Eukaryotic Cell 11: 1055-1066.

Kronstad JW, Leong SA. 1989. Isolation of two alleles of the $b$ locus of Ustilago maydis. Proceedings of the National Academy of Sciences of the United States of America 86: 978-982.

Madrigal-Rodríguez J, Villanueva-Verduzco C, Sahagún-Castellanos J, Acosta-Ramos M, Martínez ML, Espinosa ST. 2010. Production test of greenhouse hydroponic huitlacoche (Ustilago maydis Cda.). Revista Chapingo Serie Horticultura 16: 177-182.

Martínez-Espinoza AD, León C, Elizarraraz G, Ruíz-Herrera J. 1997. Monomorphic nonpathogenic mutants of Ustilago maydis. Phytopathology 87: 259-265.

Mueller AN, Ziemann S, Treitschke S, Abmann D, Doehlemann G. 2013. Compatibility in the Ustilago maydis-maize interaction requires inhibition of host cysteine proteases by the fungal effector Pit2. PLoS Pathogens 9: e1003177. doi:10.1371/journal.ppat.1003177.

Rambaut A, Drummond A. 2010. FigTree v1. 3.1. Edinburgh, Institute of Evolutionary Biology, University of Edinburgh

Romeis T, Brachmann A, Kahmann R, Kämper J. 2000. Identification of a target gene for the $\mathrm{bE} / \mathrm{bW}$ homeodomain protein complex in Ustilago maydis. Molecular Microbiology 37: 54-66.

Ronquist F, Huelsenbeck JP. 2003. MrBayes 3: Bayesian phylogenetic inference under mixed models. Bioinformatics 19: 1572-1574.

Rowell JB, DeVay JE. 1954. Genetics of Ustilago zeae in relation to basic problems of its pathogenicity. Phytopathology 44: 356-362.

Sabbagh SK, Diagne-Lèye G, Naudan M, Rioux CP. 2010. Solopathogenic strain formation strongly differs among Ustilaginaceae species. FEMS Microbiology Letters 305: 121-127.

Schulz B, Banuett F, Dahl M, Schlesinger R, Schäfer W, Martin T, Herskowitz I, Kahmann R. 1990. The $b$ alleles of Ustilago maydis, whose combinations program pathogenic development, code for polypeptides containing a homeodomain-related motif. Cell 60: 295-306.

Shutleff CM. 1980. Compendium of Corn Disease. Saint Paul, American Phytopathological Society.

Snetselaars KM, Mims CW. 1993. Infection of maize stigmas by Ustilago maydis: light and electron microscopy. Phytopathology 83: 843-850.

Stoll M, Piepenbring M, Begerow D, Oberwinkler F. 2003. Molecular phylogeny of Ustilago and Sporisorium species (Basidiomycota, Ustilaginales) based on internal transcribed spacer (ITS) sequences. Canadian Journal of Botany 81: 976-984

Tamura K, Stecher G, Peterson D, Filipski A, Kumar S. 2013. MEGA6: molecular evolutionary genetics analysis version 6.0. Molecular Biology and Evolution 30: 2725-2729.

Thompson JD, Higgins DG, Gibson TJ. 1994. Clustal W: improving the sensitivity of progressive multiple sequence alignment through sequence weighting, position-specific gap penalties and weight matrix choice. Nucleic Acids Research 22: 4673-4680.

Tracy WF, Vargas C, Zepeda L, Pataky JK, Chandler MA. 2007. Production and marketing of huitlacoche. In: Janick J, Whipkey A. (eds.) Issues in new crops and new uses. Alexandria, ASHA Press. p. 233-236.

Zhang Z, Schwartz S, Wagner L, Miller W. 2000. A greedy algorithm for aligning DNA sequences. Journal of Computational Biology 7: 203-214. 